\title{
Explaining Asset Prices with External Habits and Wage Rigidities in a DSGE Model.
}

\author{
Harald Uhlig*
}

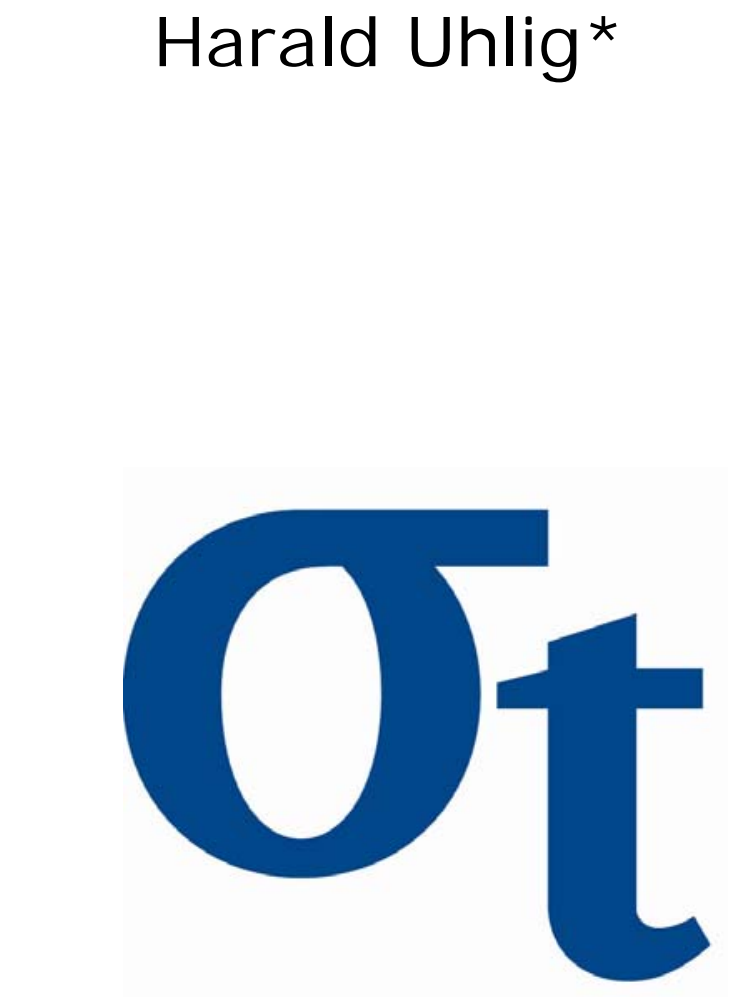

* Humboldt Universität zu Berlin, Germany Deutsche Bundesbank, CentER and CEPR

This research was supported by the Deutsche Forschungsgemeinschaft through the SFB 649 "Economic Risk". 


\title{
Explaining Asset Prices with External Habits and Wage Rigidities in a DSGE Model.*
}

\author{
Harald Uhlig \\ Humboldt Universität zu Berlin \\ Deutsche Bundesbank, CentER and CEPR \\ This version: January 15, 2007
}

\footnotetext{
${ }^{*}$ This was written for the 2006 AEA session E3 "Habit Persistence and the Macroeconomy", chaired by Martin Uribe and Stephanie Schmitt-Grohe. The discussant was Fatih Guvenen. Address: Prof. Harald Uhlig, Humboldt Universität zu Berlin, Wirtschaftswissenschaftliche Fakultät, Spandauer Str. 1, 10178 Berlin, GERMANY. email: uhlig@wiwi.hu-berlin.de, phone: +49-30-2093 5626, fax: +49-30-2093 5934, home page http://www.wiwi.hu-berlin.de/wpol/. This research was supported by the Deutsche Forschungsgemeinschaft through the SFB 649 "Economic Risk". I am grateful to Fatih Guvenen for useful feedback.
} 


\section{Explaining Asset Prices with External Habits and Wage Rigidities in a DSGE Model. ${ }^{1}$}

This paper is a progress report on understanding the relationship between prices and allocations of risks on financial markets versus macroeconomic choices and allocations. In this paper, I investigate the scope of a model with exogenous habit formation - or "catching up with the Joneses", see Abel (1990) - to generate the observed equity premium as well as other key macroeconomic facts. Along the way, I derive restrictions for four out of eight parameters for a rather general preference specification of habit formation by imposing consistency with long-run growth, the leisure share, the aggregate Frisch elasticity of labor supply, the observed risk-free rate, and the observed Sharpe ratio.

The high reward for holding risk on financial markets implies that the stochastic discount factor of the marginal investor shows large fluctuations, which are highly correlated with aggregate risk. Models with a representative agent - to which this paper belongs - therefore require, that risk aversion, measured appropriately, must be high. That alone, however is not enough: when economic choices are endogenous, agents typically have the possibility to insulate the risk-sensitive dimensions of their preferences against aggregate risk.

Thus, the literature on generating both asset pricing facts as well as macroeconomic facts within one model has increasingly pointed to labor market frictions as possibly important for a joint explanation. Endogenous labor

\footnotetext{
${ }^{1}$ This research was supported by the Deutsche Forschungsgemeinschaft through the SFB 649 "Economic Risk". I am grateful to Fatih Guvenen for useful feedback.
} 
supply decisions on a frictionless labor market provide agents with an insurance device against fluctuations in consumption. This insurance possibility then renders these models incapable of generating high Sharpe ratios or equity premia, unless additional frictions on labor markets such as separated labor markets or wage rigidities are introduced, see e.g. Lettau and Uhlig (2000), Boldrin, Christiano and Fisher (2001), Guvenen (2003) or Uhlig (2006). Furthermore, wage rigidities have recently been emphasized as key to understanding aggregate fluctuations more generally, see e.g. Hall (2005), Shimer (2005) and Blanchard and Gali (2005). I shall therefore pay particular attention to the role of wage rigidities here.

\section{The model}

I shall use small letters to denote the choices of an individual agent, and capital letters to denote economy-wide averages per agent. Production is organized by firms, hiring labor and renting capital from households at a market wage $W_{t}$ and a market dividend $D_{t}$. I assume that production is competitive and follows a Cobb-Douglas production function,

$$
Y_{t}=K_{t-1}^{\theta}\left(e^{z_{t}} N_{t}\right)^{1-\theta}
$$

and therefore

$$
W_{t}=(1-\theta) Y_{t} / N_{t}, D_{t}=\theta Y_{t} / K_{t-1}
$$

Technology $z_{t}$ evolves according to a random walk with drift,

$$
z_{t}=\gamma z_{t-1}+\epsilon_{t}
$$

where I shall assume in the linearized version of the model, that $\epsilon_{t}$ is normally iid with standard deviation $\sigma_{\epsilon}$. 
A representative agent has preferences given by

$$
U=E\left[\sum_{t=0}^{\infty} \beta^{t} \frac{\left(\left(c_{t}-H_{t}\right)\left(A+\left(l_{t}-F_{t}\right)^{\nu}\right)\right)^{1-\eta}-1}{1-\eta}\right]
$$

where $c_{t}$ and $l_{t}$ denote consumption and leisure, and $\beta, \nu, \eta, A$ are parameters, satisfying $\nu>0, \eta>\nu /(\nu+1)$ to assure monotonicity and concavity on the domain. She is endowed with initial capital $k_{-1}$ and one unit of time per period, which can be used as labor or leisure. She maximizes these preferences over choices of investment, consumption and labor, taking as given the "exogenous habits" of $H_{t}$ for consumption and $F_{t}$ for leisure as well as real wages $W_{t}$ for labor and dividends $D_{t}$ for providing capital to firms,

$$
\begin{aligned}
1 & =n_{t}+l_{t} \\
c_{t}+x_{t} & =D_{t} k_{t-1}+W_{t} n_{t} \\
k_{t} & =\left(1-\delta+g\left(\frac{x_{t}}{k_{t-1}}\right)\right) k_{t-1}
\end{aligned}
$$

Define

$$
\tilde{\delta}=\delta+e^{\gamma}-1
$$

I assume that the adjustment cost function $g(\cdot)$ satisfies

$$
g(\tilde{\delta})=\tilde{\delta}, g^{\prime}(\tilde{\delta})=1, g^{\prime \prime}(\tilde{\delta})=-\frac{1}{\xi}
$$

for some $\xi>0$, see Jermann (1998). The exogenous habits evolve according to

$$
\begin{aligned}
H_{t} & =e^{\gamma}\left((1-\zeta) \chi C_{t-1}+\zeta H_{t-1}\right) \\
F_{t} & =(1-\phi) \psi L_{t-1}+\phi F_{t-1}
\end{aligned}
$$

where $C_{t}$ and $L_{t}$ are aggregate average levels of consumption and leisure. There is an extra constant $\gamma$ in the specification for $H_{t}$ which is tied to the 
productivity growth rate in (3). I do this for algebraic simplicity. Exogenous habits give rise to externalities, which may be corrected with taxes, see Ljungqvist and Uhlig (2000): I shall ignore this issue here.

I assume that labor markets are demand constrained. The usual firstorder condition of the agent for supplying labor would be

$$
W_{t}^{f}=\frac{\partial u / \partial l}{\partial u / \partial c}=\nu \frac{c_{t}-H_{t}}{A\left(l_{t}-F_{t}\right)^{1-\nu}+l_{t}-F_{t}}
$$

at the friction-free wage $W_{t}^{f}$. Due to some unmodelled friction, I assume that not all labor supply reaches the market. I assume that the steady state supply of labor is fixed at some exogenously imposed level below the level of the frictionless economy. Locally around the steady state, households are therefore willing to supply labor at the going market wage, assumed to be governed by

$$
W_{t}=\left(e^{\gamma} W_{t-1}\right)^{\mu}\left(W_{t}^{f}\right)^{1-\mu}
$$

This is a real wage ridity as recently postulated by e.g. Hall (2005) and Shimer (2005). The particular specification here follows Blanchard and Gali (2005). Note that the frictionless scenario is included as a special case for $\mu=0$.

An equilibrium is defined in the usual way, except for imposing that labor markets are demand-constrained. In particular, individual choices will coincide with aggregate choices, e.g. $c_{t}=C_{t}$, and I shall now drop the distinction between the two. One can show that there is a steady state in the productivity-detrended variables $\tilde{C}_{t}=C_{t} / \exp \left(z_{t-1}\right)$ and similarly $\tilde{k}_{t}, \tilde{H}_{t}$, $\tilde{W}_{t}, \tilde{W}_{t}^{f}, \tilde{Y}_{t}$ and the stationary variables $L_{t}, F_{t}, N_{t}$. I denote this steady state with bars.

Assuming that $t$ counts quarters, I shall set $\delta=0.015, \gamma=0.0075$, $\theta=0.33$, as is common, implying a nonstochastic growth rate of $3 \%$ per 
year. I shall also impose on my choice of preferences parameters, that they are consistent with a nonstochastic return of $\bar{R}=1.01$ and a share of time spent as leisure as $\bar{L}=2 / 3$. With this, compute e.g.

$$
\frac{\bar{X}}{\bar{Y}}=\frac{\tilde{\delta} \theta}{\bar{R}-1+\delta}=0.30 \text { and } \kappa=\frac{1-\bar{L}}{(1-\theta) \bar{L}}\left(1-\frac{\bar{X}}{\bar{Y}}\right)=0.52
$$

which will be useful below. The eight preference parameters $(A, \beta, \eta, \nu, \chi, \zeta, \psi, \phi)$ shall now be constrained by two assumptions already made and by two further observations: the Frisch elasticity of labor supply and the Sharpe ratio.

\section{Constraints on preferences}

\subsection{Macroeconomic constraints}

Consider a more general preference specification with a per-period felicity function $u\left(c_{t}-H_{t}, l_{t}-F_{t}\right)$. Impose that leisure is constant along the balanced growth path. This implies that

$$
u\left(\left(c_{t}-H_{t}, l_{t}-F_{t}\right)=\frac{\left(\left(c_{t}-H_{t}\right) v\left(l_{t}-F_{t}\right)\right)^{1-\eta}-1}{1-\eta}\right.
$$

for some function $v(\cdot)$ and up to the intercept and scaling. For a loglinear approximation, the derivatives of $\log v(\cdot)$ and $\log v^{\prime}(\cdot)$ around the steady state characterize this function sufficiently. In my specification, I set $v\left(l_{t}-F_{t}\right)=A+\left(l_{t}-F_{t}\right)^{\nu}$. Thus, my habit preference specification is general up to a second-order approximation, subject to obeying the balanced growth condition for preferences of the form (15). For algebraic convenience, define

$$
\alpha=A(1-\psi)^{-\nu} \bar{L}^{-\nu}, \tilde{\eta}=\frac{\eta}{1-\chi}, \tilde{\nu}=\frac{\nu(1-\eta)}{(1+\alpha)(1-\psi)}
$$


Comparing the marginal utility of leisure with its marginal opportunity costs yields

$$
\frac{1-\psi}{1-\chi} \frac{1+\alpha}{\nu} \geq \frac{\bar{C}}{\bar{W}^{f} \bar{L}}=\kappa
$$

where the inequality ought to be strict in order to induce the labor market to be demand-constrained, and where the latter equality derives from the firstorder conditions of the firm and steady state substitutions. This equation delivers my first constraint

$$
\frac{1}{\eta} \geq 1+\kappa \frac{\tilde{\nu}}{\tilde{\eta}}
$$

as well as, equivalently,

$$
\alpha \geq \kappa \nu \frac{1-\chi}{1-\psi}-1
$$

Let $\tau$ be the Frisch elasticity of labor supply, which I shall treat here as the elasticity of desired labor supply with respect to a change in the frictionless wage, holding marginal utility of consumption constant. Given preference parameters $\chi$ and $\eta$, define

$$
\Upsilon(\chi, \eta)=\frac{1-\bar{N}}{\tau \bar{N}}-\left(2-\frac{1}{\eta}\right) \frac{1}{(1-\chi) \kappa}
$$

After some calculation, I obtain the second constraint

$$
\nu=1-(1-\psi) \Upsilon(\chi, \eta)
$$

I will use this equation to calculate the implied value for $\nu$. Note that $\nu>0$ for all $\psi \in[0,1), \chi \in[0,1)$ and $\eta>1$, if $\tau>\underline{\tau}=(1-\bar{N}) \kappa /(\bar{N}(\kappa+1))$.

\subsection{Asset pricing constraints}

Let $R_{t+1}$ be the return on some asset between period $t$ and $t+1$. The Lucas asset pricing equation is

$$
1=\beta E_{t}\left[\frac{\lambda_{t+1}}{\lambda_{t}} R_{t+1}\right]
$$


where

$$
\lambda_{t}=u_{c, t}=\left(c_{t}-H_{t}\right)^{-\eta}\left(A+\left(l_{t}-F_{t}\right)^{\nu}\right)^{1-\eta}
$$

For the nonstochastic growth path (although not for the average risk-free rate in the stochastic economy), this implies the third constraint

$$
\beta=e^{\eta \gamma} \bar{R}^{-1}
$$

Define the news,

$$
\epsilon_{\lambda, t+1}=\log \left(\lambda_{t+1}\right)-E_{t}\left[\log \left(\lambda_{t+1}\right)\right]
$$

and define similarly $\epsilon_{R, t+1}, \epsilon_{c, t+1}$ and $\epsilon_{l, t+1}$. Let $\sigma_{\lambda}, \sigma_{R}, \rho_{\lambda, R}$ be the standard deviations of $\epsilon_{\lambda, t+1}$ and $\epsilon_{R, t+1}$ and their correlation. Define similarly $\sigma_{c}, \sigma_{l}$ and $\rho_{c, l}$. I assume homoskedasticity throughout. Let $r_{t}^{f}$ be the logarithm of the risk-free return from $t$ to $t+1$. Define the Sharpe ratio

$$
S R=\frac{\log E_{t}\left[R_{t+1}\right]-r_{t}^{f}}{\sigma_{R}}
$$

Assuming joint normality in (22) delivers

$$
S R=-\rho_{\lambda, r} \sigma_{\lambda}
$$

see Lettau-Uhlig (2002) for a detailed derivation. The maximally possible Sharpe ratio therefore is

$$
S R=\sigma_{\lambda}
$$

In asset pricing, distributional assumptions and the choice of numerical approximation methods are not innocuous, see e.g. Judd and Guo (2001) and Weitzmann (2005). Also, nonlinearities are key for Campbell and Cochrane (1999) to explain a number of facts jointly. Finally, the linear habit preference specification in (4) can generate an ill-defined maximization problem, unless 
the shock process is sufficiently restricted. Resolving these issues is beyond the scope of this paper. Rather, these remarks shall serve as a caveat for the log-linear approach pursued here. This approach has the advantage of being well-understood and imposing a tight discipline on the exercise.

To a log-linear approximation,

$$
\epsilon_{\lambda, t+1}=-\tilde{\eta} \epsilon_{c, t+1}+\tilde{\nu} \epsilon_{l, t+1}
$$

Equation (28) therefore implies a quadratic equation in $\tilde{\eta}$ and $\tilde{\nu}$,

$$
\left(\frac{(1-\chi) S R}{\eta \sigma_{c}}\right)^{2}=1-\rho_{c, l} \frac{\sigma_{l}}{\sigma_{c}} \frac{\tilde{\nu}}{\tilde{\eta}}+\left(\frac{\sigma_{l}}{\sigma_{c}} \frac{\tilde{\nu}}{\tilde{\eta}}\right)^{2}
$$

which is my forth constraint. Note that $\tilde{\nu}=0$, if $\tilde{\eta} \sigma_{c}=S R$, which is the benchmark case of no influence of leisure on asset pricing, and holds for separable preferences, $\eta=1$.

\section{$3 \quad$ Numerical strategy and results}

\subsection{Parameterization}

The observations above constrain the preference parameters $(\alpha, \beta, \eta, \nu, \chi, \psi)$, leaving two degrees of freedom. I use $(\chi, \psi)$ to parameterize this solution manifold. I assume that the demand-constraint on labor markets is moderate and I shall therefore treat (18) as equality. Replace $\tilde{\nu} / \tilde{\eta}$ in (30) with (18), imposing equality there. Given $\chi$, equation (30) is a quadratic equation in $\eta$, which generally has two solutions. I shall use the solution to the right of the minimum. If $\chi$ is sufficiently small - which means $\chi \leq 0.97$ in the numerical calculations - this results in $\eta>1$. Calculate $\Upsilon(\eta, \chi)$ and therefore $\nu$ in (21). Find $\alpha$ from (19) with equality. Find $\beta$ from (24), where I allow $\beta>1$.

Check $\nu>0, \eta>\nu /(\nu+1)$ and $\alpha>-1$ or start over with a new $(\chi, \psi)$. 
For equation (30) I shall use $S R=0.15$ as the quarterly Sharpe ratio. On an annual basis, this implies a Sharpe ratio of approximately 0.3 , which is lower than the usual 0.5 ratio quoted in the literature, but appropriate here, given the definition in term of log-returns. I use $\sigma_{c}=0.67 \%, \sigma_{l}=0.45 \%$ and $\rho_{c, l}=-0.33$, calculated from taking first-differences of the log-series rather than innovation standard deviations, which would be more appropriate. For the Frisch elasticity of labor supply, I use a value of 3.

The remaining free parameters are thus $\left(\chi, \psi, \zeta, \phi, \xi, \mu, \sigma_{\epsilon}\right)$. I always rescale $\sigma_{\epsilon}$ so that the HP-filtered volatility of ouput equals 2 . For the other parameters, I conducted a hyperparameter search, using a grid for each of these six parameters. The goal is to find parameter constellations which endogenously deliver a quarterly Sharpe ratio of 0.15 , a risk-free rate volatility of $1.7 \%$, a ratio of consumption volatility to output volatility of 0.47 , a ratio of investment volatility to output volatility of 3.95 and a ratio of labor volatility to output volatility of 1.03. I minimize a criterion function, imposing a weight of 1 on squared deviations for all values, except using a weight of 50 for the Sharpe ratio. I exclude solutions with explosive behaviour (which may happen with $\beta>1$ ) or other numerical problems.

For the grid, I use $0, .3, .6, .8, .9, .95, .97$ for both habit level parameters $\chi$ and $\psi$. I use $0, .1, .2, .3, .5, .7, .9$ for both habit persistence parameters $\phi$ and $\zeta$. I set $\xi^{-1}=0, .05,0.1,0.15,0.2,0.5,1$. Finally, I use $\mu=.2, .3, .35, .4, .6, .8$. The overall minimum has been found at $\psi=0.97, \chi=0.8, \zeta=\phi=0, \xi^{-1}=$ $0.1, \mu=0.35$ implying $\eta=7, \nu=1.5, \beta=1.04, \alpha=4.3$, while the minimum without sticky wages was found at $\psi=0.9, \chi=0.97, \zeta=0, \phi=0.7, \xi^{-1}=$ 0.2. Apparently, habit persistence is not needed (i.e. $\zeta=\phi=0$ ), if sticky wages are allowed for. The required degree of wage stickiness is moderate. When wages are restricted to be flexible, it seems important to allow for 
considerable persistence in leisure habit.

At the overall minimum, the model simulations deliver $S R=0.25$ on an annualized basis, $\sigma_{r}=1.84, \sigma_{c} / \sigma_{y}=0.85, \sigma_{n} / \sigma_{y}=0.81, \sigma_{x} / \sigma_{y}=2.22$, $\sigma_{\epsilon} / 0.172=1.63, \operatorname{corr}(\mathrm{c}, \mathrm{y})=0.91, \operatorname{corr}(\mathrm{n}, \mathrm{y})=0.73$ and $\operatorname{corr}(\mathrm{x}, \mathrm{y})=0.84$, where e.g. $\sigma_{c}$ now denotes the volatility of HP-filtered consumption. These numbers are close to the data. The model delivers both the observed Sharpe ratio as well as a moderate risk-free rate volatility. Consumption fluctuates more in the model than what is observed in the data, while investment fluctuates less. Also, I need the fluctuations in the productivity innovations to be about $60 \%$ higher than what is traditionally assumed. This may simply reflect the necessity for shocks that have not been included here yet.

At the flexible wage minimum, the model simulations deliver $S R=0.08$ on an annualized basis, $\sigma_{r}=2.12, \sigma_{c} / \sigma_{y}=0.31, \sigma_{n} / \sigma_{y}=0.66, \sigma_{x} / \sigma_{y}=2.69$, $\sigma_{\epsilon} / 0.172=1.37, \operatorname{corr}(\mathrm{c}, \mathrm{y})=0.72, \operatorname{corr}(\mathrm{n}, \mathrm{y})=0.94$ and $\operatorname{corr}(\mathrm{x}, \mathrm{y})=0.62$. Now, the Sharpe ratio is just a quarter of what it ought to be, despite giving this particular target a high weight in the criterion function. Apparently, it is hard to match the observed Sharpe ratio without giving up considerably on other quantitative implications of the model.

In summary, a DSGE model with (exogenous and lagged) habits in both leisure and consumption, but not necessarily with additional persistence, is well capable of matching the observed asset market facts as well as macro facts, provided one allows for moderate real wage stickiness and provided one allows for sufficient curvature on preferences, as dictated by the asset market observations. Without wage stickiness, delivery on both the asset pricing implications as well as the macroeconomic implications seems to be much harder. 


\section{References}

[1] Abel, Andrew. "Asset Prices under Habit Formation and Catching Up with the Joneses," American Economic Review, American Economic Association,1990. 80(2), pp. 38-42.

[2] Blanchard, Olivier and Gali, Jordi. "Real Wage Rigidities and the New Keynesian Model,". Working paper 05-27, MIT, Department of Economics, 2005.

[3] Boldrin, Michele, Christiano, Lawrence J. and Fisher, Jones D.M. "Habit Persistence, Asset Returns, and the Business Cycle." American Economic Review, 2001. 91(1), pp. 149-66.

[4] Campbell, John Y. and John H. Cochrane (1999). "By Force of Habit: A Consumption-Based Explanation of Aggregate Stock Market Behavior." Journal of Political Economy, 1999. 107(2), pp. 205-51.

[5] Guvenen, Fatih. "A Parsimonious Macroeconomic Model for Asset Pricing: Habit Formation or Cross-Sectional Heterogeneity?". Draft, University of Texas-Austin (2003).

[6] Hall, Robert F. "Employment Fluctuations with Equilibrium Wage Stickiness." American Economic Review, 2005. 95(1), pp. 50-65.

[7] Jermann, U.J. "Asset pricing in production economies." Journal of Monetary Economics, 1998. 41, pp. 257-275.

[8] Judd, Kenneth L. and Guu, Sy-Ming. "Asymptotic methods for asset market equilibrium analysis." Economic Theory, 2001. 18, pp. 127-157. 
[9] Lettau, Martin and Uhlig, Harald. "Can Habit Formation Be Reconciled with Business Cycle Facts?" Review of Economic Dynamics, 2000. 3(1), pp. 79-99.

[10] Lettau, Martin and Uhlig, Harald. "The Sharpe Ratio and Preferences: A Parametric Approach." Macroeconomic Dynamics, 2002. 6(2), pp. $242-65$.

[11] Ljungqvist, Lars and Uhlig, Harald. "Tax Policy and Aggregate Demand Management under Catching Up with the Joneses." American Economic Review, 2000. 90(3), pp. 356-366.

[12] Shimer, Robert. "The Cyclical Behavior of Equilibrium Unemployment, Vacancies and Wages: Evidence and Theory." American Economic Review, 2005. 95(1), pp. 25-49.

[13] Uhlig, Harald (2006), "Macroeconomics and Asset Markets: some Mutual Implications." Draft, Humboldt Universität zu Berlin, 2005.

[14] Weitzmann, Martin L. "Risk, Uncertainty, and Asset-Pricing 'Puzzles'." Draft, Harvard University, 2005. 


\section{SFB 649 Discussion Paper Series 2007}

For a complete list of Discussion Papers published by the SFB 649, please visit http://sfb649. wiwi. hu-berlin.de.

001 "Trade Liberalisation, Process and Product Innovation, and Relative Skill Demand" by Sebastian Braun, January 2007.

002 "Robust Risk Management. Accounting for Nonstationarity and Heavy Tails" by Ying Chen and Vladimir Spokoiny, January 2007.

003 "Explaining Asset Prices with External Habits and Wage Rigidities in a DSGE Model." by Harald Uhlig, January 2007.

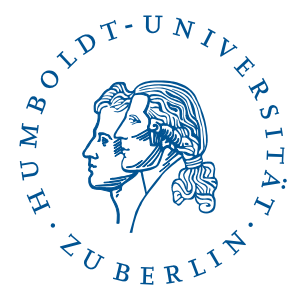

